\title{
Ground Deformation Propagation Above a TBM tunnel in Canada - A Case Study
}

\author{
Ahmed Fahmy', Mrinmoy Kanungo ${ }^{1}$ \\ ${ }^{1} 30$ Forensic Engineering \\ 40 University Ave, Toronto, Canada \\ Afahmy@30fe.com; mkanungo@30fe.com
}

\section{Extended Abstract}

Localized ground deformations were observed in the vicinity of an advancing tunnel boring machine TBM in Canada. A detailed post-failure investigation program was carried out in an effort to identify the root cause(s) of the observed damage.

The study includes documentation of the visible damage in and surrounding the tunnel, examination of the field instrumentation data recorded during tunnel advancement, detailed review of the tunnel design calculations and finite element simulations, examination of the construction activities, and assessment of the suitability of the tunnelling technique for the subject subsurface conditions.

The results of this study show that a combination of factors resulted in the observed ground settlement including ineffective tail void grouting, localized over-excavation and improper maintenance of face pressure in the rather adverse soil conditions. The results also highlight the importance of proper quality control during tunnelling including proper documentation of excavated soil volume records during construction. Discussion of settlement propagation in light of the different site constraints and environmental factors are presented. Finally, observations made during the remediation of the observed deformations are provided. 